\title{
13
}

\section{REMUNERATION FOR EXECUTIVES AND MANAGEMENT}

\author{
Brian Kearney
}

Executive remuneration that is well structured and clearly linked to the strategic objectives of the organisation is important for long-term stability and growth. Executive directors who contribute to the long-term success of that organisation should be rewarded appropriately and on a transparent basis as stakeholders want to see remuneration being used effectively to attract and incentivise executives as well as fostering a culture for sustainable growth.

Not all organisations will have a remuneration committee (RemCom) in place; however, the work of this committee is integral to achieving the board-approved objectives. People play an enormous role in achieving those objectives, and it is vitally necessary that how they are remunerated supports the achievement of those objectives and does not conflict with them. Aside from the performance of executive directors and senior management at board meetings and presentations (non-executive) directors have their most insightful view of their management teams as a result of being on their remuneration committee.

DOI: $10.4324 / 9781003201182-15$ 
It is important that when the RemCom is part of the organisation's structure, that it has a complete, appropriate, and regularly reviewed terms of reference (ToR). For these cases it has been assumed in the following that the RemCom ToR empowers the RemCom to make any awards under board-approved incentive/bonus plans. For those organisations without a RemCom, this work becomes part of the general board of directors' remit. So given the above, what should directors be asking about executive remuneration?

\section{Early in the strategy cycle}

In many ways this is probably the most critical stage as it can set the tone and parameters for all aspects of executive remuneration. Fundamental errors and/or misunderstandings at this stage can have lasting effects on the organisation's performance and stakeholder relations, particularly for shareholders and employees.

- Has the RemCom been asked for its views on the capabilities and appropriateness of the executive team (Execs) to be charged with developing and presenting the new/updated strategy for the organisation?

- How do the execs' remuneration packages and their structure compare with comparable businesses in your sector, and in particular those at a similar stage in the strategy cycle (even if historically)?

- In what respects do the historic incentivisation awards for the execs suggest they are achievers and capable of delivering the strategy, including significant change if required?

- How has the RemCom aligned the execs' incentivisation with the achievement of the strategy objectives?

- How has the RemCom assessed whether the incentivisation elements are sufficient - if awarded - to make a difference to the relevant execs?

- How well distributed are the packages at the various senior levels - CEO, C-suite, and senior management, and reflective of the likely contributors to success in achieving the strategy?

- Do the incentive plans encourage teamwork or individual contribution or initiative, and which or what combination does the RemCom consider most appropriate for the strategy under consideration? 


\section{Development and implementation of long-term incentive plans (LTIPs)}

There is a wide range of considerations involved in designing and implementing an effective LTIP with implications for all the organisation's stakeholders. Many organisations use external expertise to help them develop their LTIPs, and the RemCom would be well advised to have access to the appropriate expertise on design, market norms, taxation, and employee contracts. The resulting LTIP needs to be appropriate to the specific needs of your particular organisation and not blindly follow your adviser's advice, however expert.

- How does the LTIP support the organisation's strategy?

- Can the LTIP support variation in that strategy?

- How does the LTIP align executives' interests with those of shareholders and the wider environmental, social, and governance (ESG) stakeholders?

- What compromises have been made in having regard to these - sometimes competing - interests, to arrive at a practical and effective LTIP?

- How has the senior management been involved in the design of the LTIP?

- Are the timescales and duration of the LTIP compatible with the successful implementation of the organisation's strategy?

- At the end of the LTIP life, how can the RemCom show that potential awards to management will be fair in the context of shareholder rewards and the organisation's ESG commitments?

\section{Start of budget cycle}

While of relatively short duration, the budget period is hopefully a further step forward in the achievement of the organisation's strategy. Inevitably there will be many things to be achieved in the next 12 months, but it is part of the RemCom's role to ensure the remuneration packages of the senior team help keep that team focused on achieving the longer-term goal that this budget is a step towards. Although pay rises or other pay adjustments may be set out in board-approved budgets, there should not be an automatic process to grant such remuneration changes but rather prior proper consideration. 
- Relative to success in achieving last year's targets, which members of the management team appear capable of delivering the coming year's targets?

- Relative to the budget targets, how reasonable do the changes in targets look relative to performance targets set for individual team members?

- What apparent contradictions or inconsistencies are there between the execs' targets for the year and those set out in the organisation's strategy?

- Do any of the exec team's personal targets suggest their skill base may need to be enhanced, or even may need to be replaced in the short term?

- Are the proposed packages for execs appropriate, rational, and have they had some degree of market validation?

\section{Annual incentivisation awards}

Hopefully, there is sufficient interaction between the chair of the RemCom, the CEO, and the board chair so that the RemCom is not dealing with major surprises. In particular, it is not helpful if the outcome of this RemCom meeting comes as a major surprise to the board or the senior employees. The CEO should be able to support the recommendation for awards for their direct reports, and such other executives as has been agreed. These recommendations should be consistent with the non-executive director's knowledge of the individual's performance during the year. It goes without saying that the CEO, while it is reasonable to put forward their case for their own bonus, should not be present for the discussion around approval or otherwise of their award.

- How have awards been distributed over the various senior levels?

- Why have any particular levels been disproportionately rewarded or penalised?

- Have there been significant amendments made by the RemCom to the CEO's award recommendations in respect of the CEO's direct reports?

- To what extent has the RemCom amended awards which might have been made on the basis of measurable metrics alone? 
- What is the proportion of achievable bonuses that have been awarded at the various levels, and how has the RemCom rationalised that in the context of the organisation's performance?

- Has the RemCom had appropriate opportunity to discuss the CEO's proposed award in their absence (and that of an executive chairman) if relevant?

\section{Vesting of long-term incentivisation awards}

In theory, having put an effective LTIP in place, it should then be a simple matter for the RemCom to formally approve occurrence of the individual's accrued right to the award. However, virtually all LTIP schemes give the RemCom varying degrees of discretion over the actual awards, both as regards timing and quantum. Equally, during the life of the scheme, the makeup of execs may change and/or beneficiaries may move from one category to another. RemCom decisions are just as relevant now to ensure that strategy and LTIP objectives can continue to be achieved and appropriate individuals make up the executive team. Even if there has been no change in the make-up of the team, the RemCom should ask itself periodically whether that executive team still has the skills and capabilities needed to achieve those objectives. If not, the RemCom should bring it to the attention of the board, if necessary without executives present, with such recommendations as it believes necessary.

- In what respects and to what extent has the RemCom exercised its discretion on vesting of the LTIP?

- How has the RemCom shown that the objectives of the LTIP have been achieved?

- Does the executive team continue to be appropriate?

- How do the organisation's various remuneration schemes ensure that it does not now face a potential critical loss of skills and experience with the finalisation of the LTIP? 